\title{
Omega-3 fatty acids for depression in adults (Protocol)
}

\author{
Appleton KM, Perry R, Sallis HM, Ness AR, Churchill R
}

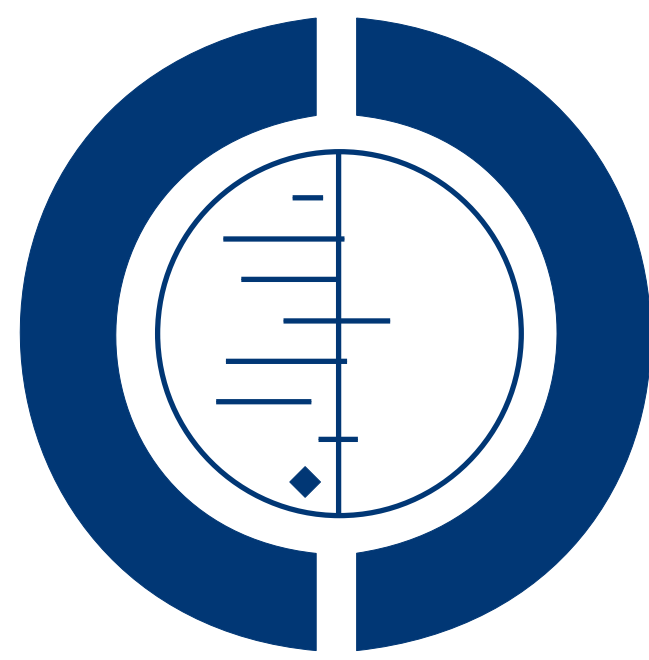

THE COCHRANE COLLABORATION $^{\circledR}$

This is a reprint of a Cochrane protocol, prepared and maintained by The Cochrane Collaboration and published in The Cochrane Library 2014, Issue 5

http://www.thecochranelibrary.com

\section{WILEY}


TABLE OF CONTENTS

HEADER . . . . . . . . . . . . . . . . . . . . . . . . . . . . . . . . . . . . 1

ABSTRACT . . . . . . . . . . . . . . . . . . . . . . . . . . . . . . . . . . . . . . . . . .

BACKGROUND . . . . . . . . . . . . . . . . . . . . . . . . . . . . . . . . . . . .

OBJECTIVES . . . . . . . . . . . . . . . . . . . . . . . . . . . . . . . . . . . . . . .

METHODS . . . . . . . . . . . . . . . . . . . . . . . . . . . . . . . . . . . . . .

ACKNOWLEDGEMENTS . . . . . . . . . . . . . . . . . . . . . . . . . . . . . . . . . . . . . . . .

REFERENCES . . . . . . . . . . . . . . . . . . . . . . . . . . . . . . . . . . . . . . 8

APPENDICES . . . . . . . . . . . . . . . . . . . . . . . . . . . . . . . . . . . . . . 11

WHAT'S NEW .. . . . . . . . . . . . . . . . . . . . . . . . . . . . . . . . . . . . . . . . . . . . . . 13

CONTRIBUTIONS OF AUTHORS . . . . . . . . . . . . . . . . . . . . . . . . . . . . . . . . . . . 13

DECLARATIONS OF INTEREST . . . . . . . . . . . . . . . . . . . . . . . . . . . . . . . . . . . . . .

SOURCES OF SUPPORT . . . . . . . . . . . . . . . . . . . . . . . . . . . . . . . . . . . . . . . . . . . . . . 


\title{
[Intervention Protocol]
}

\section{Omega-3 fatty acids for depression in adults}

\author{
Katherine M Appleton ${ }^{1}$, Rachel Perry ${ }^{2}$, Hannah M Sallis ${ }^{3,4}$, Andrew R Ness ${ }^{5}$, Rachel Churchill ${ }^{3}$ \\ ${ }^{1}$ Department of Psychology, Bournemouth University, Poole, UK. ${ }^{2}$ The NIHR Biomedical Research Unit at the University of Bristol \\ and the University Hospitals Bristol NHS Foundation Trust in Nutrition, Diet and Lifestyle, School of Oral and Dental Sciences, \\ University of Bristol, Bristol, UK. ${ }^{3}$ Centre for Academic Mental Health, School of Social and Community Medicine, University of \\ Bristol, Bristol, UK. ${ }^{4}$ MRC Integrative Epidemiology Unit, School of Social and Community Medicine, University of Bristol, Bristol, \\ UK. ${ }^{5}$ NIHR Biomedical Research Unit in Nutrition, Diet and Lifestyle, Level 3, University Hospitals Bristol Education Centre, Bristol, \\ UK
}

Contact address: Katherine M Appleton, Department of Psychology, Bournemouth University, Poole House, Fern Barrow, Poole, BH12 5BB, UK. k.appleton@bournemouth.ac.uk.

Editorial group: Cochrane Depression, Anxiety and Neurosis Group.

Publication status and date: Amended to reflect a change in scope (see 'What's new'), published in Issue 5, 2014.

Citation: Appleton KM, Perry R, Sallis HM, Ness AR, Churchill R. Omega-3 fatty acids for depression in adults. Cochrane Database of Systematic Reviews 2014, Issue 5. Art. No.: CD004692. DOI: 10.1002/14651858.CD004692.pub3.

Copyright (C) 2014 The Cochrane Collaboration. Published by John Wiley \& Sons, Ltd.

\begin{abstract}
A B S T R ACT
This is the protocol for a review and there is no abstract. The objectives are as follows:

To assess the effects of n3 polyunsaturated fatty acids (n3PUFAs) (also known as omega-3 fatty acids) compared with comparator (e.g. placebo, anti-depressant treatment, standard care, no treatment, wait-list control) for major depressive disorder in adults.
\end{abstract}

\section{B A C K G R O U N D}

\section{Description of the condition}

Major depressive disorder (MDD) is characterised by: depressed mood; markedly diminished pleasure or interest in all activities; significant weight loss or weight gain, or decrease or increase in appetite; insomnia or hypersomnia; psychomotor agitation or retardation; fatigue or lethargy; feelings of worthlessness or inappropriate guilt; disruptions to concentration and decision-making; and recurrent thoughts of death (APA 2013). Diagnosis is achieved by: the presence of four or more symptoms (as above) plus depressed mood or markedly diminished pleasure or interest in all activities, for a consecutive period of two weeks; significant distress or impairment in functioning as a result of the symptoms; and an inability to attribute symptoms to the physiological effects of a substance or another medical condition (APA 2013). MDD is currently estimated to affect approximately $7 \%$ of western populations, with resulting impact both at an individual and a societal level (APA 2013). MDD can be highly debilitating; affect all areas of an individual's life; can be difficult to treat, with a high rate of recurrence; and often exists in combination with other conditions and disorders, such as cardiovascular disease and anxiety disorders (APA 2013). Recent figures (2011) by the World Health Organization estimate major depressive disorders to account for 3\% of global ill health in terms of disability-adjusted life years (WHO 2014), and projections for 2030 suggest an increase to $6 \%$ to $7 \%$ (WHO 2014). Given this increasing trend, there is an urgent need for effective treatments and strategies for prevention.

Omega-3 fatty acids for depression in adults (Protocol)

Copyright $\odot 2014$ The Cochrane Collaboration. Published by John Wiley \& Sons, Ltd. 


\section{Description of the intervention}

One emerging potential treatment for MDD exists in the family of $\mathrm{n} 3$ polyunsaturated fatty acids (n3PUFAs), also known as omega3 fatty acids.

n3PUFAs are a family of polyunsaturated fatty acids, named as such due to the positioning of the first double carbon bond on the third atom from the methyl end of the acyl chain. All members of the family are derived from the parent fatty acid 18:3n3 (Alphalinolenic Acid (ALA)), via desaturation and elongation. ALA however can not be synthesised by humans, and thus must be obtained from the diet (Haag 2003; Ruxton 2005). Longer chain n3PUFAs can be formed in humans, but biological conversion is slow and inefficient, making diet an important source for these fatty acids as well (Ma 1995). Dietary sources of ALA include certain nuts and seeds, such as walnuts, flaxseed and rapeseed (canola) oil. Dietary sources of the longer n3PUFAs eicosapentaenoic acid (EPA) and docosahexaenoic acid (DHA) include fatty fish, some white fish, shellfish and other seafood such as seaweed, and certain eggs and animal products dependent on the animal's diet (BNF 1999; James 2000; Ruxton 2005; Simopolous 1999).

Links between n3PUFAs and MDD were first suggested following recognition of a drastic reduction in the dietary consumption of n3PUFAs in recent decades (Simopolous 1999). Coupled with this reduction in $\mathrm{n} 3 \mathrm{PUFA}$ intakes, intakes of $\mathrm{n} 6$ long chain polyunsaturated fatty acids (n6PUFAs) have considerably increased. Closely related to the n3PUFAs, n6PUFAs (named from the positioning of the first double bond on the sixth carbon atom from the methyl end of the acyl chain) are derived from the parent essential fatty acid 18:2n6 (Linoleic Acid (LA)), and for synthesis, share the same desaturases and elongases as n3PUFAs. N3PUFAs and n6PUFAs thus compete for synthesis from their parent fatty acids. Dietary sources of LA and all n6PUFAs include plant and vegetable seeds and oils, as found in margarines and the majority of processed foods (James 2000; Simopolous 1999). Our traditional diet is thought to have contained approximately equal amounts of energy from n3PUFAs and n6PUFAs (Simopolous 1999). By comparison, a current western diet is estimated to contain approximately 5 to 20 times more energy from n6PUFAs than from n3PUFAs (Gregory 2000; Simopolous 1999).

Early work investigating population consumption levels of n3PUFAs and n3PUFA rich foods, such as fish, suggest clear links with population levels of MDD and other psychiatric conditions (Hibbeln 1998; Noaghiul 2003; Peet 2004), and repeated studies since have also found similar associations. Within countries, n3PUFA intakes have been negatively associated with depressive illness (e.g. Silvers 2002; Tanskanen 2001). In clinical studies, low levels of n3PUFAs have been found in individuals diagnosed with MDD (e.g. Edwards 1998; Peet 1998) and depressive disorders (e.g. Garland 2007), and reporting high levels of depressed mood (e.g. Mamalakis 2002; Mamalakis 2006) compared to controls. Continuous relationships between n3PUFA status and depressive symptoms have also been found (e.g. Edwards 1998). In ran- domised controlled trials (RCTs), beneficial effects of supplementation with n3PUFAs compared to placebo have been reported for MDD (e.g. Nemets 2002; Su 2003) and depressive disorders (e.g. Frangou 2006; Stoll 1999).

\section{How the intervention might work}

The positive effects of $\mathrm{n} 3$ PUFAs on depressive illness are thought to occur as a result of changes to cell membrane structure and function, impacting particularly on cell communication, inflammatory processes and neurotransmitter activities (Haag 2003; James 2000; Ruxton 2005). Further details are available in Appendix 1. Disruptions to and abnormal cell signalling, inflammatory processes and neurotransmitter system activities have been implicated in MDD (Parker 2006; Stahl 2008).

\section{Why it is important to do this review}

N3PUFAs are known to be important in brain development and function, and have been linked to depression in a variety of studies, see Appendix 2. Not all studies, however, report beneficial effects (see Appendix 2), and reviews and meta-analyses clearly demonstrate considerable variability between studies (e.g. Appleton 2006; Appleton 2008b; Appleton 2010; Lin 2007; Parker 2006; Smith 2011; Stahl 2008). Meta-analyses reveal some small benefit of n3PUFAs for depressive disorders (Appleton 2006; Lin 2007), but investigations of the considerable heterogeneity also suggest differential effects of $\mathrm{n} 3$ PUFAs, dependent primarily on severity of depressive symptoms at baseline (Appleton 2010). Sensitivity analyses based on severity of depressive symptoms at baseline suggest no benefits of n3PUFAs for individuals with mild depressive symptoms or without diagnosis of depression, but also provide some evidence of benefits in individuals with severe depressive symptoms or with depressive diagnoses (Appleton 2010). These findings suggest a possible benefit of n3PUFAs for MDD. This review will investigate a role of n3PUFAs as a treatment for MDD. Various reviews of other treatments for MDD and other depressive disorders are available. A recent search of The Cochrane Library revealed 98 completed reviews or reviews in progress focusing on treating or preventing depression. The majority of these reviews investigate pharmacological (e.g. antidepressant) or psychological (e.g. cognitive behavioural therapy) therapies for depressive conditions, or focus on specific clinical populations, e.g. stroke patients or patients with diabetes mellitus. Only two of these reviews include n3PUFAs, both focussing on antenatal and postnatal depression. One review investigates 'dietary supplements for preventing postnatal depression' (Miller 2013), and includes one study of n3PUFAs. This study found no significant preventive impact of n3PUFAs on the presence of postnatal depression. The other review (Dennis 2013) includes two trials investigating the use of $\mathrm{n} 3 \mathrm{PUFAs}$ for antenatal depression, and reports a beneficial effect 
on depression in one trial and no benefits in the other. One further review also focuses on a herbal treatment (St John's Wort) for depression (Linde 2008), but the active component of this plantbased treatment is unrelated to n3PUFAs or other fatty acids.

\section{O B J E C T I VES}

To assess the effects of $\mathrm{n} 3$ polyunsaturated fatty acids (n3PUFAs) (also known as omega-3 fatty acids) compared with comparator (e.g. placebo, anti-depressant treatment, standard care, no treatment, wait-list control) for major depressive disorder in adults.

\section{METHODS}

\section{Criteria for considering studies for this review}

\section{Types of studies}

Only randomised controlled trials (RCTs) will be included, as the best study design for assessing an intervention. All RCTs will be included regardless of quality, but measures of risk of bias will be recorded. We will exclude observational and case-control studies. We will include cross-over RCTS, although only the first study phase of cross-over trials will be used in analyses, to avoid carryover effects.

Cluster RCTs will be included in primary analyses, where the cluster will act as the unit of investigation. We think cluster and especially cross-over RCTs are unlikely to be used in this field. Our aim is to include as many relevant studies as possible to avoid limitations and bias, while recording possible confounding and investigating this where numbers allow.

\section{Types of participants}

\section{Participant characteristics}

Studies will be included regardless of participant demographics (e.g. gender, age, country of residence), although only studies involving adults (18 years and over) will be included.

\section{Diagnosis}

We will only include studies that enrolled participants with a diagnosis of major or unipolar depressive disorder from a trained professional or using a validated rating scale, or studies that include a subgroup of these individuals. If a subgroup is used, only the data from the subgroup will be included in the review. If data from diagnosed and non-diagnosed individuals are mixed, these studies and these data will not be included. Studies that enrolled participants without MDD, but with an alternative depressive disorder, e.g. bipolar disorder, postpartum depression (APA 2013) will not be included. We will exclude studies that describe a diagnosis of MDD that is given only during or in relation to pregnancy.

Studies will be included regardless of participant medication. Studies involving participants with concomitant medication will be included due to the high likelihood of use in the MDD population (APA 2013), and a desire to make the review as generalisable as possible. Medication will be recorded as part of the review and investigated in subgroup analyses if possible.

\section{Comorbidities}

We will include studies regardless of the use of participants with other comorbid conditions (physical conditions, e.g. CHD or psychiatric conditions, e.g. anxiety). Studies involving participants with comorbid conditions will be included due to the high likelihood of existing comorbidities in the MDD population (APA 2013), and a desire to make the review as generalisable as possible. Investigation of effects due to existing comorbidities will be undertaken in subgroup analyses, where possible dependent on available data.

\section{Setting}

Studies will be included regardless of setting, provided a clinical diagnosis has been provided.

\section{Types of interventions}

\section{Experimental intervention}

Studies will be included if they use an exposure of n3PUFAs as a sole or adjunctive therapy. Studies will be included regardless of: the source of n3PUFA provided (pure ALA, EPA, DHA or any combination of these, fish, flaxseed, rapeseed, etc); the dose of $\mathrm{n} 3$ PUFA or duration of supplementation; and the mode of provision (i.e. supplement capsules, supplemented foods), but records of these differences will be made. Studies will be included in the main review if details of type of n3PUFA, dose, and ratio are not available, as mechanisms for action remain unknown. Studies with a 'lead-in' phase to allow for spontaneous remission or placebo responding in participants will be included, and use of the 'lead in' phase will be recorded.

\section{Comparator intervention}

Studies will be included regardless of the comparator used, but a comparator must be used and all comparators will be recorded, and investigated if possible. Wait-list controls, no treatment or 
standard care will be included as comparators. Studies will be included regardless of participant medication. Studies involving participants with concomitant medication will be included due to the high likelihood of use in the MDD population (APA 2013), and a desire to make the review as generalisable as possible. Medication will be recorded as part of the review and investigated in subgroup analyses if possible.

\section{Types of outcome measures}

We will include studies that meet the above criteria, regardless of whether they report on all of the following outcomes.

\section{Primary outcomes}

1. Depressive symptomology (continuous data) - Depressive symptomology will be assessed using any continuous validated measure of depressive symptomology. The most commonly used validated rating scales are the Beck Depression Inventory (Beck 1987), the Montgomery-Asberg Depression Rating Scale (Montgomery 1979), and the Hamilton Depression Index (Hamilton 1960), but studies using other scales will also be included.

2. Adverse events - Measures of adverse events will be recorded if possible. Number and type (e.g. gastrointestinal, psychiatric) of adverse events experienced will be recorded as reported in studies. Number of individuals suffering, as opposed to number of events, will be used in analyses. Where adverse events are not reported, this will be recorded.

\section{Secondary outcomes}

3. Depressive symptomology (dichotomous data) - Depressive symptomology will also be assessed using remission or improvement as assessed using clinical diagnoses by a trained professional or a validated rating scale, where provided.

4. Quality of life (continuous data) - Quality of life will be assessed using any continuous validated measure of quality of life.

5. Failure to complete - Number of individuals leaving each study early, and reasons for early drop-out will be recorded.

\section{Timing of outcome assessment}

Where studies use multiple time points, data from the longest follow-up period only will be used for analysis. Data will be tabulated for all outcomes at all time points where assessments have been made, but only those of longest follow-up will be included in statistical analyses. Previous work suggests that effects are likely to increase over time (Calder 2003; Ruxton 2005).

\section{Search methods for identification of studies}

Suitable studies for inclusion will be identified by searching databases, international trials registers and published review articles, and by contacting authors of published trials.

\section{Electronic searches}

\section{The Cochrane Depression, Anxiety and Neurosis Review Group's Specialised Register (CCDANCTR)}

The Cochrane Depression, Anxiety and Neurosis Group (CCDAN) maintain two clinical trials registers at their editorial base in Bristol, UK: a references register and a studies based register. The CCDANCTR-References Register contains over 31,500 reports of RCTs in depression, anxiety and neurosis. Approximately 65\% of these references have been tagged to individual, coded trials. The coded trials are held in the CCDANCTR-Studies Register and records are linked between the two registers through the use of unique Study ID tags. Coding of trials is based on the EU-Psi coding manual, using a controlled vocabulary (please contact the CCDAN Trials Search Coordinator for further details). Reports of trials for inclusion in the Group's registers are collated from routine (weekly), generic searches of MEDLINE (1950-), EMBASE (1974-) and PsycINFO (1967-); quarterly searches of the Cochrane Central Register of Controlled Trials (CENTRAL) and review specific searches of additional databases. Reports of trials are also sourced from international trials registers c/o the World Health Organization's trials portal (the International Clinical Trials Registry Platform (ICTRP)), pharmaceutical companies, the handsearching of key journals, conference proceedings and other (non-Cochrane) systematic reviews and meta-analyses.

Details of CCDAN's generic search strategies (used to identify RCTs) can be found on the Group's website.

1. The CCDANCTR (Studies and References Registers) will be searched using the following terms:

(depress * or dysthymi* or "affective disorder *" or "affective symptom *" or "mood disorder*" or "mental health") AND (dha or docosahex* or

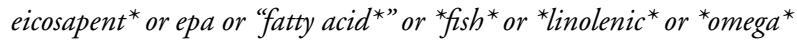
or $n-3$ or $w-3$ or *PUFA* or "cod liver oil')

2. Complementary searches will be conducted on the following bibliographic databases using relevant subject headings (controlled vocabularies) and search syntax, appropriate to each resource:

- CINAHL (Cumulative Index to Nursing \& Allied Health)

(1982 to date), search strategy listed in Appendix 3

- BIOSIS Citation Index (1969 to date)

- Web of Science (1900 to date)

There will be no restrictions on date, language or publication status applied to the searches. 
3. International trial registries will be searched via the World Health Organization's trials portal (ICTRP) and ClinicalTrials.gov to identify unpublished or ongoing studies.

\section{Searching other resources}

We will check the reference lists of all included studies and relevant systematic reviews to identify additional studies missed from the original electronic searches.

We will contact authors of relevant publications (reports of included studies) and principle investigators of currently registered trials for information on unpublished or ongoing studies or to request additional trial data.

\section{Data collection and analysis}

We will download search results into Endnote. Selected studies will be downloaded into RevMan (RevMan 2012). We will provide details of all work undertaken and number of potentially-eligible studies at all steps of the search and selection process.

\section{Selection of studies}

Two review authors (RP, HS) will independently screen for inclusion the titles and abstracts of all the studies we identify as a result of the search, and code them as 'retrieve' (eligible or potentially eligible/unclear) or 'do not retrieve'. We will retrieve the potentially-relevant full-text study reports/publication and two review authors (RP, HS) will independently screen the full-text and identify studies for inclusion, and identify and record reasons for exclusion of the ineligible studies. We will resolve any disagreement through discussion or, if required, we will consult a third person (KA). We will identify and exclude duplicate records, and we will collate multiple reports that relate to the same study, so that each study rather than each report is the unit of interest in the review. Titles or abstracts which may be relevant, but where relevance is not clear, will be included in the list and obtained. Articles in foreign languages will be obtained and translated where necessary, as may be the case, particularly for unpublished or preliminary data. We will record the selection process in sufficient detail to complete a PRISMA flow diagram and 'Characteristics of excluded studies' table.

\section{Data extraction and management}

We will use a data collection form, developed specifically for this work, to extract study characteristics and outcome data, which has been piloted on at least one study in the review. Two review authors (HS and KA or RP) will extract study characteristics and outcome data from included studies. We will extract the following study characteristics.
1. Methods: study design, total duration of study, details of any 'lead in' period, use of several study centres, study location, study setting, and date of study.

2. Participants: N, mean age, age range, gender, severity of condition, diagnostic criteria, inclusion criteria, and exclusion criteria, withdrawals.

3. Interventions: intervention, comparator, concomitant medications, and comorbidities.

4. Outcomes: primary and secondary outcomes specified and collected, and time points reported.

5. Notes: funding for trial, and notable conflicts of interest of trial authors.

Where multiple reports of the same study are available, data will be abstracted from all reports on separate data extraction forms and subsequently combined. Discordances will be resolved by independent abstraction and then discussion with a third author. Where data are unavailable as required, corresponding authors will be contacted directly for relevant information.

We will note in the 'Characteristics of included studies' table if outcome data were not reported in a usable way. We will resolve disagreements by consensus or by involving a third person (RC, AN). Two review authors (RP, HS) will transfer data into the Review Manager (RevMan 2012) file. We will double-check that data are entered correctly by comparing the data presented in the systematic review with the study reports. A second review author (KA) will spot-check study characteristics for accuracy against the trial report.

\section{Main comparisons}

- n3PUFAs versus comparator. We will conduct analyses by comparator type.

\section{Assessment of risk of bias in included studies}

Three review authors (KA, RP, HS) will independently assess the risk of bias for each study using the criteria outlined in the Cochrane Handbook for Systematic Reviews of Interventions (Higgins 2011). We will resolve any disagreements by discussion or by involving another author (RC, AN). We will assess the risk of bias according to the following domains.

1. Random sequence generation.

2. Allocation concealment.

3. Blinding of participants and personnel.

4. Blinding of outcome assessment.

5. Incomplete outcome data.

6. Selective outcome reporting.

7. Other bias.

We will judge each potential source of bias as high, low or unclear risk and provide a supporting quotation from the study report together with a justification for our judgment in the 'Risk of bias' table. We will summarise the risk of bias judgements across different studies for each of the domains listed. Where information on 
risk of bias relates to unpublished data or correspondence with a triallist, we will note this in the 'Risk of bias' table. When considering treatment effects, we will take into account the risk of bias for the studies that contribute to that outcome.

\section{Measures of treatment effect}

\section{Dichotomous data}

Depressive diagnosis or status will be recorded as provided. Dichotomous data will be collected in the form of $\mathrm{N}$ with diagnosis per intervention group. We will analyse dichotomous data as odds ratios (OR) with $95 \%$ confidence intervals (CI).

\section{Continuous data}

Depressive symptomology and quality of life will be recorded using all scales as used in each study, after ensuring comparable direction. Analyses will be conducted on data from only one scale per study. For depressive symptomology, we will use either the scale most commonly used in all studies, or the Beck Depression Inventory (Beck 1987) as the most respected of depression scales validated in depressive populations. For quality of life, we will use the scale most commonly used in all studies reporting quality of life.

Continuous data will be collected in the form of $\mathrm{N}$, mean, and standard deviation, per intervention group at baseline and at the end of each intervention, as required for meta-analysis. If data are only provided in other forms, e.g. as medians, change from baseline, we will contact study authors and request appropriate data. Data from subgroups of little relevance to the research question, e.g. groups of males and females, will be recorded as reported, and subsequently combined for analysis.

We will analyse continuous data as mean difference (MD) with 95\% CI or standardised mean difference (SMD) with 95\% CI. We will enter data presented as a scale with a consistent direction of effect. We will undertake meta-analyses only where this is meaningful, i.e. if the treatments, participants and the underlying clinical question are similar enough for pooling to make sense. We will narratively describe skewed data reported as medians and interquartile ranges. Where multiple trial arms are reported in a single trial, we will include only the relevant arms.

Data on adverse events will be reported by number of individuals suffering as opposed to number of events.

Data on failure to complete will be reported as number of individuals failing to complete each trial, and reasons given for noncompletion.

\section{Unit of analysis issues}

\section{Cross-over RCTs}

Only the first study phase of cross-over RCTs will be included in analyses. We think cross-over RCTs are unlikely to be used in this field.

\section{Cluster RCTs}

Cluster RCTs will be included in primary analyses, where the cluster will act as the unit of investigation. We think cluster RCTs are unlikely to be used in this field.

\section{Studies with multiple treatment groups}

Where studies use multiple treatment groups, each treatment group will be treated independently and included in all appropriate analyses. In these cases, the same comparator will be used for all treatment groups. Assuming individuals took part in only one treatment/comparator group, groups are independent. Data from comparison groups will be split across treatment groups for analysis. Studies where individuals are provided with multiple treatments will be considered as cross-over RCTs; however we think these sorts of studies are very unlikely to be used.

\section{Dealing with missing data}

We will contact investigators in order to verify key study characteristics and obtain missing numerical outcome data where possible (e.g. when a study is identified as abstract only). We will document all correspondence with trialists and report which trialists responded in the full review.

Standard deviations which remain missing once authors have been contacted will be imputed from standard deviation data from all other trials using the same measure for depression in the review (Furukawa 2006).

\section{Assessment of heterogeneity}

Heterogeneity will be investigated using Higgins' $\mathrm{I}^{2}$ statistic (Higgins 2002; Higgins 2004). $\mathrm{I}^{2}$ statistics and appropriate P values will be reported. Four bands of the $\mathrm{I}^{2}$ statistic will be used for interpretation, as recommended in the Cochrane Handbook (Higgins 2011). These bands are $0 \%$ to $40 \%$ : might not be important; $30 \%$ to $60 \%$ : may represent moderate heterogeneity; $50 \%$ to $90 \%$ may represent substantial heterogeneity; and $75 \%$ to $100 \%$ : considerable heterogeneity. Possible sources of heterogeneity will be identified a priori, to include publication bias, comparator used, use of n3PUFAs as a sole or adjunct therapy, presence/absence of comorbid conditions (physical and psychiatric), and risk of bias. Publication bias will be investigated using funnel plot asymmetry (Sterne 2001). Investigations of heterogeneity and sensitivity analyses on possible sources of heterogeneity will be conducted if possible. We suspect these investigations may be limited by the small number of studies likely to be identified. 


\section{Assessment of reporting biases}

Publication bias will be investigated using funnel plot asymmetry (Sterne 2001). This will only be undertaken if there are more than 10 studies available. It should be noted that publication bias is one of several possible causes of asymmetry in funnel plots.

\section{Data synthesis}

Data from trials reporting mean and standard deviation data will be combined using meta-analysis (Sterne 2001). Analyses will be conducted twice: once using only studies at low risk of bias, and once using all included studies. Intention-to-treat (ITT) data will be used, where possible, and will be sought if not publicly available. If ITT data cannot be obtained, we will use available data.

For continuous data, the standardised mean effect for all trials will be calculated using Hedges' adjusted g (Deeks 2001). Hedges' adjusted $\mathrm{g}$ is a formulation of effect size used in the SMD method that includes an adjustment to correct for small sample bias (Deeks 2001). Studies will be weighted using the inverse-variance method. Random-effects models will be used primarily to estimate the SMDs for all analyses (Deeks 2001; Egger 2001; Sterne 2001). Fixed-effect models will also be applied as sensitivity analyses. Effect sizes will be provided as means and standard deviations, and related to specific scales to allow understanding by clinicians and practitioners.

For dichotomous data, we will use the Mantel-Haenszel method. Adverse effects and failure to complete data will not be statistically summarised.

If data from a single study cannot be included in a statistical combination, this will be reported and outcomes of that single study will be reported separately.

\section{Subgroup analysis and investigation of heterogeneity}

We will conduct subgroup analyses investigating effects of n3PUFAs on MDD in:

1. individuals with comorbid conditions versus those without. This analysis will demonstrate effects due to participant characteristics which may affect treatment recommendations and outcomes. Separate analyses will be conducted using the same methods as for the main analyses, using only studies in which participants are clearly identified as having comorbid conditions, and using only studies in which participants are clearly identified as being without comorbid conditions. Studies where participants with and without comorbid conditions are mixed, and studies that do not clearly identify whether participants have comorbid conditions or not, will not be included in this analysis. Caution should be exercised in interpreting these analyses due to the increased bias in selective samples;

2. individuals receiving medications versus those without. This analysis will demonstrate effects due to participant characteristics which may affect treatment recommendations and outcomes. Separate analyses will be conducted using the same methods as for the main analyses, using only studies in which participants are clearly identified as receiving medication or adjunct therapy, and using only studies in which participants are clearly identified as not receiving medication or other adjunct therapy. Studies where participants with additional medications and therapies are mixed, and studies that do not clearly identify whether or not participants were receiving additional medication or therapies, will not be included in this analysis. Caution should be exercised in interpreting these analyses due to the increased bias in selective samples.

\section{Sensitivity analysis}

We will conduct sensitivity analyses to investigate the impact of:

1. including all studies versus only studies that are at low risk of bias. This will demonstrate the importance of the use of only those trials at low risk of bias, and the levels of confidence and caution that should be exercised in considering the analyses on all studies. Separate analyses will be conducted using the same methods as for the main analyses, using all studies, and using only the studies that are identified as being at low risk of bias. Low risk of bias will be defined as in the Cochrane Handbook (Higgins 2011);

2. using fixed-effect models as opposed to random-effects models. Random-effects models will be used for all main analyses. Fixed-effect analyses will be conducted using the same methods as for the main analyses.

\section{'Summary of findings' table}

We will provide a 'Summary of findings' table, as recommended in The Cochrane Handbook (Higgins 2011). Outcomes will include depressive symptomology and measures of adverse events. The primary comparison will be n3PUFAs versus comparator. The quality of evidence for all outcomes will be assessed using the GRADE system, as described by the Grades of Recommendation, Assessment, Development and Evaluation Working Group. This will involve consideration of within-study risk of bias (methodological quality), directness of evidence, heterogeneity, precision of effect estimates and risk of publication bias.

\section{ACKNOW LEDGEMENTS}

This work is supported by the Biomedical Research Unit (BRU), University of Bristol, UK, and Bournemouth University, UK.

\section{CRG Funding Acknowledgement:}


The National Institute for Health Research (NIHR) is the largest single funder of the Cochrane Depression, Anxiety and Neurosis Group.

\section{Disclaimer:}

The views and opinions expressed herein are those of the authors and do not necessarily reflect those of the NIHR, National Health Service (NHS) or the Department of Health.

\section{R E F E R E N C E S}

\section{Additional references}

\section{APA 2013}

American Psychiatric Association. Diagnostic and Statistical Manual of Mental Disorders, 5th Edition. Washington, DC: American Psychiatric Association, 2013.

\section{Appleton 2006}

Appleton KM, Hayward RC, Gunnell D, Peters TJ, Rogers PJ, Kessler, D, et al.Effects of $\mathrm{n} 3$ long chain polyunsaturated fatty acids on depressed mood: systematic review of published trials. American Journal of Clinical Nutrition 2006;84:1308-16.

\section{Appleton 2007}

Appleton KM, Peters TJ, Hayward RC, Heatherley SV, McNaughton SA, Rogers PJ, et al.Depressed mood and n-3 polyunsaturated fatty acid intake from fish: non-linear or confounded association?. Social Psychiatry and Psychiatric Epidemiology 2007;42:100-4.

\section{Appleton 2008a}

Appleton KM, Gunnell D, Peters TJ, Ness AR, Kessler D, Rogers PJ. No clear evidence of an associations between plasma concentrations of $n-3$ long chain polyunsaturated fatty acids and depressed mood in a non-clinical population. Prostaglandins, Leukotrienes and Essential Fatty Acids 2008; 78:337-42.

\section{Appleton 2008b}

Appleton KM, Rogers PJ, Ness AR. Is there a role for $n-3$ long-chain polyunsaturated fatty acids in the regulation of mood and behaviour? A review of the evidence to date from epidemiological studies, clinical studies and intervention trials. Nutrition Research Reviews 2008;21:13-41.

\section{Appleton 2010}

Appleton KM, Rogers PJ, Ness AR. Updated systematic review and meta-analysis of the effects of $\mathrm{n}-3$ long-chain polyunsaturated fatty acids on depressed mood. American Journal of Clinical Nutrition 2010;91:757-70.

\section{Beck 1987}

Beck AT, Steer RA. Beck Depression Inventory Manual. San Antonio, Texas: Psychological Corporation, 1987.

\section{BNF 1999}

British Nutrition Foundation. BNF Briefing paper: $n-3$ fatty acids and health. London: British Nutrition Foundation, 1999.

\section{Browne 2006}

Browne JC, Scott KM, Silvers KM. Fish consumption in pregnancy and omega-3 status after birth are not associated with postnatal depression. Journal of Affective Disorders 2006;90:131-9.

\section{Calder 2003}

Calder PC. n-3 polyunsaturated fatty acids and inflammation: from molecular biology to the clinic. Lipids 2003;38:342-52.

\section{Caughey 1996}

Caughey GE, Mantzioris E, Gibson RA, Cleland LG, James MJ. The effect on human tumor necrosis factor alpha and interleukin $1 \mathrm{~B}$ production of diets enriched in $\mathrm{n}-3$ fatty acids from vegetable oil or fish oil. American Journal of Clinical Nutrition 1996;63:116-22.

\section{Chalon 2006}

Chalon S. Omega-3 fatty acids and monoamine neurotransmission. Prostaglandins, Leukotrienes, and Essential Fatty Acids 2006;75:259-69.

\section{de la Presa Owens 1999}

De la Presa Owens S, Innis SM. Docosahexaenoic and arachidonic acid prevent a decrease in dopaminergic and serotoninergic neurotransmitters in frontal cortex caused by a linoleic and alpha-linolenic acid deficient diet in formula fed piglets. Journal of Nutrition 1999;129:2088-93.

\section{Deeks 2001}

Deeks JJ, Altman DG, Bradbrun MJ. Statistical methods for examining heterogeneity and combining results from several studies in meta-analysis. In: Egger M, Davey Smith G, Altman DG editor(s). Systematic Reviews in Health Care: Meta-analysis in Context. London: BMJ Publishing Group, 2001:285-312.

\section{Delion 1994}

Delion S, Chalon S, Herault J, Guilloteau D, Besnard JC, Durand G. Chronic dietary alpha-linolenic acid deficiency alters dopaminergic and serotonergic neurotransmission in rats. Journal of Nutrition 1994;124:2466-76. 


\section{Delion 1996}

Delion S, Chalon S, Guilloteau D, Besnard JC, Durand G. Alpha-linolenic acid dietary deficiency alters age related changes in dopaminergic and serotonergic neurotransmission in the rat frontal cortex. Journal of Neurochemsitry 1996;66:1582-91.

Dennis 2013

Dennis CL, Dowswell T. Interventions (other than pharmacological, psychosocial or psychological) for treating antenatal depression. Cochrane Database of Systematic Reviews 2013, Issue 7. [DOI: 10.1002/ 14651858.CD006795.pub3]

\section{Edwards 1998}

Edwards R, Peet M, Shay J, Horrobin D. Depletion of docosahexaenoic acid in red blood cell membranes of depressive patients. Biochemical Society Transactions 1998; 26:s142.

Egger 2001

Egger M, Davey Smith G. Principles of and procedures for systematic reviews. In: Egger M, Davey Smith G, Altman DG editor(s). Systematic Reviews in Health Care: Metaanalysis in Context. London: BMJ Publishing Group, 2001: 23-42.

\section{Ehringer 1990}

Ehringer W, Belcher D, Wassall SR, Stillwell W. A comparison of the effects of linolenic acid (18:3 omega 3) and docosahexaenoic (22:6 omega 3 ) acids on phospholipid bilayers. Chemistry and Physics of Lipids 1990;54:79-88.

Frangou 2006

Frangou S, Lewis M, McCrone P. Efficacy of ethyleicosapentaenoic acid in bipolar depression: randomised double-blind placebo-controlled study. British Journal of Psychiatry 2006;188:46-50.

\section{Furukawa 2006}

Furukawa TA, Barbui C, Cipriani A, Brambilla P, Watanabe $\mathrm{N}$. Imputing missing standard deviations in meta-analyses can provide accurate results. Journal of Clinical Epidemiology 2006;59:7-10.

Garland 2007

Garland MR, Hallahan B, McNamara M, Carney PA, Grimes H, Hibbeln JR, et al.Lipids and essential fatty acids in patients presenting with self-harm. British Journal of Psychiatry 2007;190:112-7.

\section{Gregory 2000}

Gregory J, Foster K, Tyler H, Wiseman M. National Diet and Nutritional Survey of British Adults. London: Her Majesty's Stationary Office, 2000.

\section{Grenyer 2007}

Grenyer BFS, Crowe T, Meyer B, Owen AJ, Grigonis EM, Caputi $\mathrm{P}$, et al.Fish oil supplementation in the treatment of major depression: a randomised double-blind placebocontrolled trial. Progress in Neuro-Psychopharmacology and Biological Psychiatry 2007;31:1393-6.

Haag 2003

Haag M. Essential fatty acids and the brain. Canadian Journal of Psychiatry 2003;48:195-203.

\section{Hakkarainen 2004}

Hakkarainen R, Partonen T, Haukka J, Virtamo J, Albanes $\mathrm{D}$, Lonnqvist $\mathrm{J}$. Is dietary intake of omega-3 fatty acids associated with depression?. American Journal of Psychiatry 2004;161:567-9.

\section{Hamazaki 2005}

Hamazaki K, Itomura M, Huan M, Nishizawa H. Effects of $\mathrm{w}-3$ fatty acid-containing phospholipids on blood catecholamine concentrations in healthy volunteers: a randomized, placebo controlled, double blind trial. Nutrition 2005;21:705-10.

\section{Hamilton 1960}

Hamilton M. A rating scale for depression. Journal of Neurology, Neurosurgery and Psychiatry 1960;23:56-62.

\section{Hibbeln 1998}

Hibbeln JR. Fish consumption and major depression. Lancet 1998;351:1213.

\section{Higgins 2002}

Higgins JPT, Thompson SG. Quantifying heterogeneity in a meta-analysis. Statistics in Medicine 2002;21:1539-58.

\section{Higgins 2004}

Higgins JPT, Thompson SG, Deeks JJ, Altman DG. Measuring inconsistency in meta-analyses. BMJ 2004;327: 557-60.

Higgins 2011

Higgins JPT, Green S. Cochrane Handbook for Systematic Reviews of Interventions. 5.1. Chichester, UK: The Cochrane Collaboration \& John Wiley \& Sons, Ltd, 2011.

\section{Hirashima 2004}

Hirashima F, Parow AM, Stoll AL, Demopulos CM, Damico KE, Rohan ML, et al.Omega-3 fatty acid treatment and T2 whole brain relaxation times in bipolar disorder. American Journal of Psychiatry 2004;161:1922-4.

\section{James 2000}

James MJ, Gibson RA, Cleland LG. Dietary polyunsaturated fatty acids and inflammatory mediator production. American Journal of Clinical Nutrition 2000;71:343s-8s.

Keck 2006

Keck PE Jr, Mintz J, McElroy SL, Freeman MP, Suppes T, Frye MA, et al.Double-blind, randomized, placebocontrolled trials of ethyl-eicosapentanoate in the treatment of bipolar depression and rapid cycling bipolar disorder. Biological Psychiatry 2006;60:1020-2.

Lin 2007

Lin P-Y, Su K-P. A meta-analytic review of double-blind, placebo-controlled trials of anti-depressant efficacy of omega-3 fatty acids. Journal of Clinical Psychiatry 2007;68: 1056-61.

\section{Linde 2008}

Linde K, Berner MM, Kriston L. St John's wort for major depression. Cochrane Database of Systematic Reviews 2008, Issue 4. [DOI: 10.1002/14651858.CD000448.pub3]

\section{Ma 1995}

Ma J, Folsom AR, Eckfeldt JH, Lewis L, Chambless LE, and the Atherosclerosis Risk in Communities (ARIC) Study 
Investigators. Short- and long-term repeatability of fatty acid composition of human plasma phospholipids and cholesterol esters. American Journal of Clinical Nutrition 1995;62:572-8.

\section{Mamalakis 2002}

Mamalakis G, Tornaritis M, Kafatos A. Depression and adipose essential polyunsaturated fatty acids. Prostaglandins, Leukotrienes and Essential Fatty Acids 2002;67:311-8.

\section{Mamalakis 2004}

Mamalakis G, Kiriakakis M, Tsibinos G, Kafatos A. Depression and adipose polyunsaturated fatty acids in an adolescent group. Prostaglandins, Leukotriens and Essential Fatty Acids 2004;71:289-94.

\section{Mamalakis 2006}

Mamalakis G, Kiriakakis M, Tsibinos G, Hatzis C, Flouri S, Mantzoros $\mathrm{C}$, et al.Depression and serum adiponectin and adipose omega-3 and omega- 6 fatty acids in adolescents. Pharmacology, Biochemistry and Behavior 2006;85:474-9.

\section{McNamara 2006}

McNamara RK, Richtand NM, Levant B. Omega-3 fatty acid deficiency decreases dopamine $\mathrm{D} 2$ receptor binding and increases serotonin 5-HT2A receptor binding in the adult rat prefrontal cortex. Biological Psychiatry 2006;59: $146 S$.

\section{Miller 2013}

Miller BJ, Murray L, Beckmann MM, Kent T, Macfarlane B. Dietary supplements for preventing postnatal depression. Cochrane Database of Systematic Reviews 2013, Issue 10. [DOI: 10.1002/14651858.CD009104.pub2]

\section{Miyake 2006}

Miyake Y, Sasaki A, Yokoyama T, Tanaka K, Ohya Y, Fukushima W. Risk of postpartum depression in relation to dietary fish and fat intake in Japan: the Osaka Maternal and Child Health Study. Psychological Medicine 2006;36: 1727-37.

\section{Montgomery 1979}

Montgomery SA, Asberg M. A new depression scale designed to be sensitive to change. British Journal of Psychiatry 1979;134:382-9.

\section{Nemets 2002}

Nemets B, Stahl Z, Belmaker RH. Addition of omega3 fatty acid to maintenance medication treatment for recurrent unipolar depressive disorder. American Journal of Psychiatry 2002;159:477-9.

\section{Noaghiul 2003}

Noaghiul S, Hibbeln JR. Cross-national comparisons of seafood consumption and rates of bipolar disorders. American Journal of Psychiatry 2003;160:2222-7.

\section{Parker 2006}

Parker G, Gibson NA, Brotchie H, Heruc G, Rees AM, Hadzi-Pavlovic D. Omega-3 fatty acids and mood disorders. American Journal of Psychiatry 2006;163:969-78.

\section{Peet 1998}

Peet M, Murphy B, Shay J, Horrobin D. Depletion of omega-3 fatty acid levels in red blood cell membranes of depressive patients. Biological Psychiatry 1998;43:315-9.

\section{Peet 2004}

Peet M. International variations in the outcome of schizophrenia and the prevalence of depression in relation to national dietary practices: an ecological analysis. British Journal of Psychiatry 2004;184:404-8.

\section{Rallidis 2003}

Rallidis LS, Paschos G, Liakos GK, Velissaridou AH, Anastasiadis G, Zampelas A. Dietary alpha-linolenic acid decreases $\mathrm{C}$-reactive protein, serum amyloid $\mathrm{A}$ and interleukin-6 in dyslipidaemic patients. Atherosclerosis 2003; 167:237-42.

RevMan 2012

The Nordic Cochrane Centre, The Cochrane Collaboration. Review Manager (RevMan). 5.2. Copenhagen: The Nordic Cochrane Centre, The Cochrane Collaboration, 2012.

\section{Rogers 2008}

Rogers PJ, Appleton KM, Kessler D, Peters TJ, Gunnell D, Hayward RC, et al.No effect of n-3 long chain polyunsaturated fatty acid (EPA and DHA) supplementation on depressed mood and cognitive function: a randomized controlled trial. British Journal of Nutrition 2008;99: 421-31.

\section{Ruxton 2005}

Ruxton CHS, Calder PC, Reed SC, Simpson MJA. The impact of long chain n-3 polyunsaturated fatty acids on human health. Nutrition Research Reviews 2005;18:113-29.

\section{Sawazaki 1999}

Sawazaki S, Hamazaki T, Yazawa K, Kobayashi M. The effect of docosahexaenoic acid on plasma catecholamine concentrations and glucose tolerance during long-lasting psychological stress: a double blind placebo controlled study. Journal of Nutritional Science and Vitaminology 1999; 45:655-65.

\section{Silvers 2002}

Silvers KM, Scott KM. Fish consumption and self-reported physical and mental health status. Public Health Nutrition 2002;5:427-31.

\section{Silvers 2005}

Silvers KM, Woolley CC, Hamilton FC, Watts PM, Watson RA. Randomised double-blind placebo-controlled trial of fish oil in the treatment of depression. Prostaglandins, Leukotrienes and Essential Fatty Acids 2005;72:211-8.

\section{Simopolous 1999}

Simopoulos AP. Evoluntionary aspects of omega-3 fatty acids in the food supply. Prostaglandins, Leukotrienes and Essential Fatty Acids 1999;60:421-9.

\section{Smith 2011}

Smith MA, Beilin LJ, Mori TA, Oddy WH. Essential fatty acids and mood: a systematic review of observational studies. American Journal of Food and Nutrition 2011;1: $14-27$. 


\section{Stahl 2008}

Stahl LA, Begg DP, Weisinger RS, Sinclair AJ. The role of omega-3 fatty acids in mood disorders. Current Opinion in Investigational Drugs 2008;9:57-64.

Sterne 2001

Sterne JAC, Egger M, Davey Smith G. Investigating and dealing with publication and other biases. In: Egger M, Davey Smith G, Altman DG, eds editor(s). Systematic Reviews in Health Care: Meta-analysis in Context. London: BMJ Publishing Group, 2001:189-208.

\section{Stoll 1999}

Stoll AL, Severus WE, Freeman MP, Rueter S, Zboyan HA, Diamond E, et al.Omega-3 fatty acids in bipolar disorder - a preliminary double-blind, placebo-controlled trial. Archives of General Psychiatry 1999;56:407-12.

Su 2003

Su K-P, Huang S-Y, Chiu C-C, Shen WW. Omega-3 fatty acids in major depressive disorder - a preliminary double-blind placebo-controlled trial. European Neuropsychopharmacology 2003;13:267-71.

Takeuchi 2002

Takeuchi T, Fukumoto Y, Harada E. Influence of a dietary n-3 fatty acid deficiency on the cerebral catecholamine contents, EEG and learning ability in rat. Behavioral Brain Research 2002;131:193-203.

\section{Tanskanen 2001}

Tanskanen A, Hibblen JR, Hintikka J, Haatainen K, Honkalampi K, Viinamaki K. Fish consumption, depression, and suicidality in a general population. Archives of General Psychiatry 2001;58:512-3.

Tappia 1997

Tappia PS, Ladha S, Clark DC, Grimble RF. The influence of membrane fluidity, TNF receptor binding, cAMP production and GTPase activity on macrophage cytokine production in rats fed a variety of fat diets. Molecular and Cellular Biochemistry 1997;166:135-43.

WHO 2014

World Health Organization. Global Burden of Disease. http://www.who.int/healthinfo/global_burden_disease/ gbd/en/index.html 1 Feb 2014.

Yao 2004

Yao JK, Magan S, Sonel AF, Gurklis JA, Sanders R, Reddy RD. Effects of omega-3 fatty acid on platelet serotonin responsivity in patients with schizophrenia. Prostaglandins, Leukotrienes and Essential Fatty Acids 2004;71:171-6.

\section{References to other published versions of this review}

\author{
Silvers 2009 (withdrawn) \\ Silvers KM, Hackett ML, Scott KM. Omega 3 fatty acids for \\ depression. Cochrane Database of Systematic Reviews 2009, \\ Issue 1. [DOI: 10.1002/14651858.CD004692.pub2] \\ * Indicates the major publication for the study
}

\section{A P P E N D I C ES}

\section{Appendix I. How the intervention might work}

The positive effects of n3PUFAs on depressive illness are thought to occur as a result of integration into the cell membrane phospholipid bilayer, resulting in changes in structure and function (Haag 2003; James 2000; Ruxton 2005). Incorporation into the cell membrane can influence the physical state of the membrane, resulting in increased fluidity and permeability (Ehringer 1990; Hirashima 2004; Tappia 1997), possibly aiding cross-cell membrane transport and communication (Haag 2003). Secondly, n3PUFAs are also thought to have effects on surrounding molecules and cell functions via enzyme activity which results in the release of fatty acids from the phospholipid bilayer to form a number of anti-inflammatory eicosanoids, prostaglandins, and leukotrienes (Calder 2003; James 2000; Ruxton 2005; Stahl 2008), and via enzyme activity of direct involvement in various neurotransmitter pathways (Haag 2003; James 2000; Ruxton 2005; Stahl 2008). Supplementation with n3PUFAs has been found to result in: reduced production of inflammatory cytokines - tumor necrosis factor alpha (TNFa), interleukin 1B, interleukin-6, C-reactive protein and serum amyloid A (Calder 2003; Caughey 1996; James 2000; Rallidis 2003); increased serotonergic and dopaminergic activity; and decreased concentrations of noradrenalin (Chalon 2006; de la Presa Owens 1999; Hamazaki 2005; Sawazaki 1999; Yao 2004). Additionally, n3PUFA-deficient diets have been associated with reduced receptor density and disruptions to neurotransmitter activity in serotonergic (de la Presa Owens 1999; Delion 1994; Delion 1996; McNamara 2006), dopaminergic (Chalon 2006; de la Presa Owens 1999; Delion 1994; Delion 1996; McNamara 2006; Takeuchi 2002), and adrenergic systems (Takeuchi 2002) compared to controls. Disruptions to and abnormal cell signalling, inflammatory processes and neurotransmitter system activities have been implicated in MDD (Parker 2006; Stahl 2008). 


\section{Appendix 2. Why it is important to do this review}

N3PUFAs have been linked to depression in a variety of epidemiological studies (Hibbeln 1998; Noaghiul 2003; Peet 2004; Silvers 2002; Tanskanen 2001); clinical studies (Edwards 1998; Garland 2007; Mamalakis 2002; Mamalakis 2006; Peet 1998); and RCTs (Frangou 2006; Nemets 2002; Stoll 1999; Su 2003).

However, several epidemiological studies have found no association between n3PUFA intake and depressive illness (e.g. Appleton 2007; Frangou 2006; Hakkarainen 2004; Miyake 2006; Stoll 1999; Su 2003). Clinical studies have reported no differences in n3PUFA levels between individuals diagnosed with MDD and controls (e.g. Browne 2006; Mamalakis 2004) and no clear associations (Appleton 2008a). Several RCTs have also reported no effects of supplementation on MDD (e.g. Grenyer 2007; Silvers 2005), depressive illness (e.g. Keck 2006) or depressed mood (e.g. Rogers 2008).

Reviews in this area clearly demonstrate considerable variability between studies (e.g. Appleton 2006; Appleton 2008b; Appleton 2010; Lin 2007; Parker 2006; Smith 2011; Stahl 2008). Meta-analyses also report considerable heterogeneity between studies ( Appleton 2006; Appleton 2010; Lin 2007). Meta-analyses reveal some small benefit of n3PUFAs for depressive disorders (Appleton 2006; Lin 2007), but investigations of the considerable heterogeneity also suggest differential effects of n3PUFAs dependent primarily on severity of depressive symptoms at baseline (Appleton 2010). Sensitivity analyses based on severity of depressive symptoms at baseline suggest no benefits of n3PUFAs for individuals with mild depressive symptoms or without diagnosis of depression, but also provide some evidence of benefits in individuals with severe depressive symptoms or with depressive diagnoses (Appleton 2010). These findings suggest a possible benefit of n3PUFAs for MDD.

\section{Appendix 3. CINAHL search strategy}

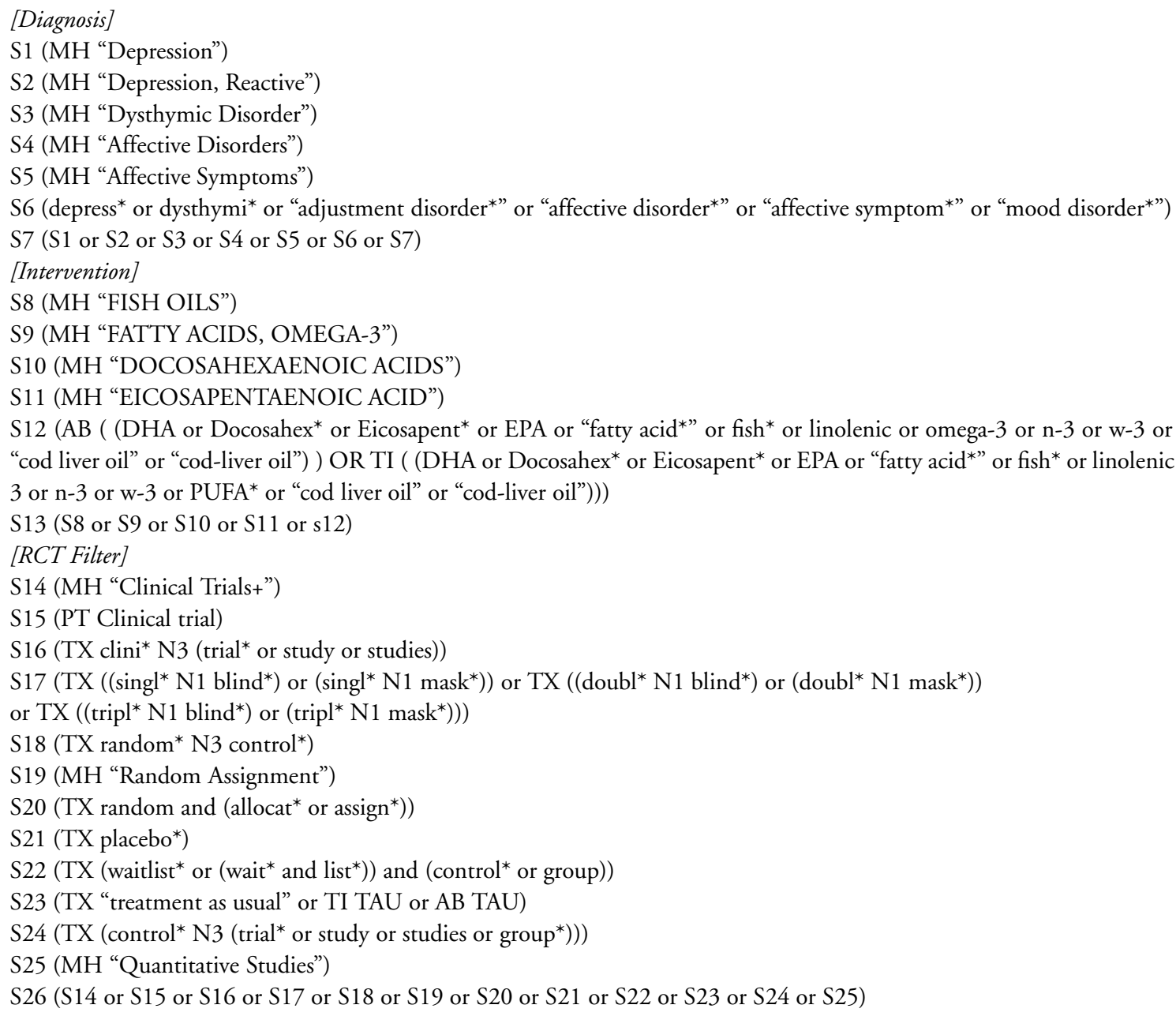

Omega-3 fatty acids for depression in adults (Protocol)

Copyright $\odot 2014$ The Cochrane Collaboration. Published by John Wiley \& Sons, Ltd. 
S27 (S7 and S13 and s26)

WHAT'S NEW

\begin{tabular}{l|l|l}
\hline Date & Event & Description \\
\hline 1 May 2014 & New citation required and major changes & This protocol replaces the withdrawn protocol Silvers 2009 (withdrawn). \\
\hline
\end{tabular}

\section{CONTRIBUTIONS OFAUTHORS}

KA wrote the protocol. All authors checked and subsequently revised this draft.

\section{DECLARATIONSOF INTEREST}

KA - None
RP - None
RC - None
AN - None
HS - None

\section{SOURCES OF SUPPORT}

\section{Internal sources}

- Bournemouth University, UK.

Researcher time

- University of Bristol, UK.

Researcher time

\section{External sources}

- No sources of support supplied 